\title{
Editorial: Investigating the human brain and muscle coupling during whole-body challenging exercise
}

\author{
Stephane Perrey * \\ EuroMov, University of Montpellier, Montpellier, France
}

Keywords: fatigue, neuroimaging, pain, sensation, peripheral feedback, perception of effort

Research into correlates and determinants (causal relationship) of fatigue has burgeoned in the past two decades. However the link between cortical activity and muscle force generation on fatigue that develops during exercise is not very well understood. While there is extensive evidence suggesting that the central nervous system can contribute to the inability of a human being to sustain a motor task, some questions are unsolved. Is fatigue a sensed variable? Could substantial progress in neurophysiological techniques have additional benefits in the understanding of fatigue? What have we learned and what happens next? Answers require likely the combined insights not only from physiologists, but of neuroscientists and psychologists. Recent research in this area presents three trends providing current interests and opinions in the field.

\section{THE PERCEPTION OF FATIGUE}

OPEN ACCESS

Edited by:

Evangelos A. Christou,

University of Florida, USA

Reviewed by:

Harsimran Singh Baweja,

San Diego State University, USA

*Correspondence:

Stephane Perrey

stephane.perrey@umontpellier.fr

Specialty section:

This article was submitted to

Exercise Physiology,

a section of the journal

Frontiers in Physiology

Received: 21 May 2015 Accepted: 28 September 2015

Published: 20 October 2015

Citation:

Perrey S (2015) Editorial: Investigating the human brain and muscle coupling during whole-body challenging exercise. Front. Physiol. 6:285. doi: 10.3389/fphys.2015.00285
The first issue concerns the fatigue state with respect to changes in motor performance. The picture of fatigue that emerges is the conscious perception of a sensation of fatigue according to the phenotypic traits of the individuals mediated by environment and culture (Boullosa and Nakamura, 2013). In this respect, muscle fatigue modifies the ratings of perceived exertion (RPE) required to maintain task performance and reduces exercise tolerance independently from metabolic stress (Marcora et al., 2008). During the fatigue state, brain is able to sense exercise intensity and environmental conditions. In this context, exercise-induced pain is proposed as a likely candidate contributing to the sensation of fatigue in exercise performance (Mauger, 2013). Athletes' attitudes toward pain and the cognitive strategies they use while experiencing pain, may be reflected in their pain tolerance levels and their performance.

The role of a second sensation brought by exercise with respect to pain is the sense of effort. The latter is centrally generated and accompanies peripheral sensory feedback on self-selected exercise intensity. While, subjective awareness of effort required to perform an exercise task can be dissociated from the physical discomfort induced by various types of exercise (e.g., sub-maximal constant-effort cycling and brief sub-maximal and maximal cycling bouts in Christian et al., 2014). Here, at the core of many integrative models explaining fatigue with the central nervous system highly involved in exercise performance regulation (Boullosa and Nakamura, 2013) is the concept of fatigue as a sensation or emotion (Noakes, 2012).

\section{THE EXERCISING BRAIN}

Probably, development of fatigue state is dependent on the short-term reorganization of the prefrontal-motor network during exercise. Methods to challenge this idea are now emerging. 
Imaging the brain during increased physiological demand (i.e., exercise) with sufficient ecological validity (Mauger, 2013) is a rapidly growing field.

Advances in functional neuroimaging techniques of the human brain with behavioral outcomes, such as electroencephalography (EEG) and near-infrared spectroscopy (NIRS), helped to generate first valuable data in human exercise research. Increased muscle recruitment as revealed by electromyographic activity was accompanied by a higher brain cortical activity (measured by EEG) localized mainly in the primary motor cortices during unilateral isometric knee extensions (Abeln et al., 2013). This bilateral activation of motor cortex observed also in NIRS study (Derosière et al., 2014) during graded unimanual force production may be caused by the occurrence of interhemispheric inhibition (i.e., mirror activity of the passive limb during unilateral movement). Other brain regions, e.g., the mid/anterior insular cortex, were demonstrated by means of EEG to play an important role in the mediation of motor performance by exerting influence on motor regions (Hilty et al., 2011). To compensate for diminished force production capability, brain regions governing the motor behavior increase their couplings (Jiang et al., 2012). These studies indicate that coordination among brain regions is an important factor underpinning the behavioral manifestations of fatigue.

Another brain region involved in motor performance is the prefrontal cortex (PFC). The PFC involved in motivational and decision-making processes is an important candidate for acting as relay station in the central fatigue related network regulating central command. Development of fatigue at the highest force output during intermittent isometric upper limb contractions was attributed to reduced muscle oxygen availability rather than impaired prefrontal oxygenation (Bhambhani et al., 2014). Conversely, elevated metabolic demands during incremental cycling task (Racinais et al., 2014) affect both PFC and muscle oxygenation levels, and in turn, muscle recruitment responses. Indeed, the well-recognized PFC deoxygenation signature during the last stages of incremental exercise (quadradic trend, in Rooks et al., 2010) occurs concomitantly with an increased

\section{REFERENCES}

Abeln, V., Harig, A., Knicker, A., Vogt, T., and Schneider, S. (2013). Brain-imaging during an isometric leg extension task at graded intensities. Front. Physiol. 4:296. doi: 10.3389/fphys.2013.00296

Bhambhani, Y., Fan, J. L., Place, N., Rodriguez-Falces, J., and Kayser, B. (2014). Electromyographic, cerebral, and muscle hemodynamic responses during intermittent, isometric contractions of the biceps brachii at three submaximal intensities. Front. Physiol. 5:190. doi: 10.3389/fphys.2014.00190

Boullosa, D. A., and Nakamura, F. Y. (2013). The evolutionary significance of fatigue. Front. Physiol. 4:309. doi: 10.3389/fphys.2013.00309

Christian, R. J., Bishop, D. J., Billaut, F., and Girard, O. (2014). The role of sense of effort on self-selected cycling power output. Front. Physiol. 5:115. doi: 10.3389/fphys.2014.00115

De Pauw, K., Roelands, B., Marusic, U., Tellez, H. F., Knaepen, K., and Meeusen, R. (2013). Brain mapping after prolonged cycling and during recovery in the heat. J. Appl. Physiol. 115, 1324-1331. doi: 10.1152/japplphysiol.00633.2013

Derosière, G., Alexandre, F., Bourdillon, N., Mandrick, K., Ward, T. E., and Perrey, S. (2014). Similar scaling of contralateral and ipsilateral cortical responses electromyographic activity (Racinais et al., 2014). Overall, these findings suggest that during whole body exercise but not during static exercise, there is inadequate delivery of oxygen to the brain. This could lead to altered PFC responses (i.e., executive function and cognitive control processes) to exercise.

In recent years, noninvasive brain stimulation techniques like transcranial direct current stimulation (tDCS) have also gained popularity owing to its potential to explore the neural processes underlying motor functions (Mauger, 2013). Recent reports used tDCS to produce beneficial effects on motor performance by attempting to reverse fatigue (Muthalib et al., 2013; Williams et al., 2013). Further development combining neuroimaging techniques with tDCS is needed in order to track reorganization changes across different levels of the nervous system during fatigue development.

\section{POST-EXERTION BRAIN FATIGUE}

Investigating the post-exercise recovery strategies could shed light on the contributions of the brain into the control of muscle force with fatigue development. Regarding the post-exercise recovery period, little or no data are available concerning central processes of performance (De Pauw et al., 2013). Assisting in brain recovery was recently highlighted (Minett and Duffield, 2014; Rattray et al., 2015). Main contributors on recovery processes in the brain following exercise are carbohydrate intake, temperature change, sleep quality, and modulation of brain activity with tDCS. Providing a window on the dynamics of human brain functioning under physiological conditions after exercise combining EEG with NIRS techniques is the next phase.

Recent evidences hold that the central nervous system ensures a significant role in the control of muscle force production in an extremely flexible manner both during exercise and in recovery. The potential utilities of complimentary and novel techniques (EEG-NIRS-tDCS) to understand cortical brain function during and after exercise in healthy humans should guide our future works.

during graded unimanual force generation. Neuroimage 85(Pt 1), 471-477. doi: 10.1016/j.neuroimage.2013.02.006

Hilty, L., Langer, N., Pascual-Marqui, R., Boutellier, U., and Lutz, K. (2011). Fatigue-induced increase in intracortical communication between $\mathrm{mid} /$ anterior insular and motor cortex during cycling exercise. Eur. J. Neurosci. 34, 2035-2042. doi: 10.1111/j.1460-9568.2011.07909

Jiang, Z., Wang, X. F., Kisiel-Sajewicz, K., Yan, J. H., and Yue, G. H. (2012). Strengthened functional connectivity in the brain during muscle fatigue. Neuroimage 60, 728-737. doi: 10.1016/j.neuroimage.2011.12.013

Marcora, S. M., Bosio, A., and de Morree, H. M. (2008). Locomotor muscle fatigue increases cardiorespiratory responses and reduces performance during intense cycling exercise independently from metabolic stress. Am. J. Physiol. Regul. Integr. Comp. Physiol. 294, R874-R883. doi: 10.1152/ajpregu.00678.2007

Mauger, A. R. (2013). Fatigue is a pain-the use of novel neurophysiological techniques to understand the fatigue-pain relationship. Front. Physiol. 4:104. doi: 10.3389/fphys.2013.00104

Minett, G. M., and Duffield, R. (2014). Is recovery driven by central or peripheral factors? A role for the brain in recovery following intermittent-sprint exercise. Front. Physiol. 5:24. doi: 10.3389/fphys.2014.00024 
Muthalib, M., Kan, B., Nosaka, K., and Perrey, S. (2013). Effects of transcranial direct current stimulation of the motor cortex on prefrontal cortex activation during a neuromuscular fatigue task: an fNIRS study. Adv. Exp. Med. Biol. 789, 73-79. doi: 10.1007/978-1-4614-7411-1_11

Noakes, T. D. (2012). Fatigue is a brain-derived emotion that regulates the exercise behavior to ensure the protection of whole body homeostasis. Front. Physiol. 3:82. doi: $10.3389 /$ fphys.2012.00082

Racinais, S., Buchheit, M., and Girard, O. (2014). Breakpoints in ventilation, cerebral and muscle oxygenation, and muscle activity during an incremental cycling exercise. Front. Physiol. 5:142. doi: 10.3389/fphys.2014. 00142

Rattray, B., Argus, C., Martin, K., Northey, J., and Driller, M. (2015). Is it time to turn our attention toward central mechanisms for post-exertional recovery strategies and performance? Front Physiol. 6:79. doi: 10.3389/fphys.2015. 00079

Rooks, C. R., Thom, N. J., McCully, K. K., and Dishman, R. K. (2010). Effects of incremental exercise on cerebral oxygenation measured by near-infrared spectroscopy: a systematic review. Prog. Neurobiol. 92, 134-150. doi: 10.1016/j.pneurobio.2010.06.002

Williams, P. S., Hoffman, R. L., and Clark, B. C. (2013). Preliminary evidence that anodal transcranial direct current stimulation enhances time to task failure of a sustained submaximal contraction. PLOS ONE 8:e81418. doi: 10.1371/journal.pone.0081418

Conflict of Interest Statement: The author declares that the research was conducted in the absence of any commercial or financial relationships that could be construed as a potential conflict of interest.

Copyright (c) 2015 Perrey. This is an open-access article distributed under the terms of the Creative Commons Attribution License (CC BY). The use, distribution or reproduction in other forums is permitted, provided the original author(s) or licensor are credited and that the original publication in this journal is cited, in accordance with accepted academic practice. No use, distribution or reproduction is permitted which does not comply with these terms. 